\title{
Correlation of the local and the macroscopic properties of high-temperature superconductors
}

\author{
Alexey Menushenkov*,I, Alexey Kuznetsov ${ }^{\mathrm{I}}$, Roman Chernikov ${ }^{\mathrm{I}}$, Andrey Ivanov, Vladimir Sidorov ${ }^{\mathrm{I}}$ \\ and Konstantin Klementiev ${ }^{\text {II }}$ \\ I National Research Nuclear University "MEPhI” Kashirshskoe shosse, 31, 115409 Moscow, Russian Federation \\ II CELLS - ALBA Carretera BP, 1413, 08290 Barcelona, Spain
}

Received July 26, 2010; accepted July 29, 2010

\section{EXAFS / High temperature superconductivity /} Double-well potential / Electron-phonon coupling

\begin{abstract}
Temperature dependent comparative EXAFS studies of $\mathrm{Ba}_{1-x} \mathrm{~K}_{x} \mathrm{BiO}_{3}$ superconducting oxides and copper based superconductors with hole $\left(\mathrm{La}_{2-x} \mathrm{Sr}_{x} \mathrm{CuO}_{4}\right)$ and electron $\left(\mathrm{Nd}_{2-x} \mathrm{Ce}_{x} \mathrm{CuO}_{4-\delta}\right)$ doping showed the common dynamical local structure nonuniformity observed as the double-well potential of oxygen ion oscillations. Obtained data evidence that these local structure nonuniformity arises from the local dynamic charge ordering due to different electronic structure of the neighbouring $\mathrm{BiO}_{6}$ or $\mathrm{CuO}_{n}$ $(n=4,6)$ complexes in perovskite-like lattice. Based on the experimental results, the phenomenological description was proposed which allowed us to establish the correlations between the local and the macroscopic properties and to explain the insulator-metal phase transition and the appearance of the superconductive state with both hole and electron doping of parent $\mathrm{BaBiO}_{3}, \mathrm{La}_{2} \mathrm{CuO}_{4}$ and $\mathrm{Nd}_{2} \mathrm{CuO}_{4}$ insulators. The presented results makes clear the role of electron-phonon coupling in the mechanism of high temperature superconductivity.
\end{abstract}

\section{Introduction}

Though superconductivity in $\mathrm{BaPb}_{1-x} \mathrm{Bi}_{x} \mathrm{O}_{3}$ (BPBO) was discovered significantly earlier [1] than in cuprates [2], the question of the nature of superconducting state in this oxides as well as in cognate system $\mathrm{Ba}_{1-x} \mathrm{~K}_{x} \mathrm{BiO}_{3}$ (BKBO) is still unsolved. Moreover in spite of extraordinary efforts for over 20 years, no universally accepted theory exists with regard to the origin of high temperature superconductivity (HTSC) in cuprates and up to now it is not clear if the mechanism of superconductivity is the same or different in these two classes of the superconducting oxides so as in hole-doped and electron-doped cuprates.

The both classes have perovskite-like lattice with $\mathrm{CuO}_{n}$ $(n=4,5,6)$ or $\mathrm{Bi}(\mathrm{Pb}) \mathrm{O}_{6}$ complexes joined by the common oxygen ions. In bismuthates, the intersection of octa-

* Correspondence author (e-mail: menushen@htsc.mephi.ru) hedral complexes in the three crystallographic directions determines their three-dimensional cubic structure. In cuprates the $\mathrm{CuO}_{n}$ complexes are joined in $\mathrm{CuO}_{2}$ planes, which makes the two-dimensional layer structure of copper-oxides. Because of strong hybridisation of covalent $\mathrm{Bi}(\mathrm{Pb}) 6 s, \mathrm{Cu} 3 d-\mathrm{O} 2 p_{\sigma}$ bonds, the above mentioned complexes are the most tightly bound items of the perovskitelike structure.

In contrast to conventional superconductivity where phonons lead to the formation of Cooper pairs, in HTSC oxides, the role of electron-phonon coupling has long been neglected. However during last time the discussion concerning the role of the lattice vibrations in the high temperature superconductivity recommences [3]. This renewed activity is connected with long list of new experimental data. Strong electron-phonon coupling was observed for both hole- and electron-doped HTSC compounds in neutron or X-ray scattering experiments and in tunneling spectra (see, for example, [4] and references therein). Recent high resolution angle-resolved photoemission (ARPES) measurements have found an oxygen isotope effect in the dispersion kink at the half-breathing phonon mode, hinting at an important role of oxygen vibrations inside the superconducting $\mathrm{CuO}_{2}$ plane [5]. Since the above phonon modes are essentially local in their character, very important information may be extracted by the extended X-ray absorption fine structure (EXAFS) technique which is the most suitable for study of the local structure peculiarities.

Considerable quantity of the EXAFS evidences for the existence of low-temperature local structure anomalies of $\mathrm{CuO}_{2}$ plane in hole-doped HTSC-cuprates was reported up to date [6-12]. The in-plane $\mathrm{Cu}-\mathrm{O}$ bonds show unconventional broadening at low temperature, which is connected with dynamic lattice instability related to superconductivity [11, 12]. Moreover this instability was interpreted as anharmonicity-induced multiphonon processes [13] and as oxygen ion vibration in a double-well potential in some investigations [7-10].

All points that lattice vibrations play a crucial role in the superconductivity of cuprates. However it is not clear whether the electron-phonon coupling is responsible for the pairing and strength of the electron-pairs binding, or it 
provides the ability to carry a superconductive current attributed to the phase stiffness $\varrho_{s}$ [14].

For the first time the existence of the double-well vibration potential was experimentally observed by us in the superconductive oxides $\mathrm{Ba}_{1-x} \mathrm{~K}_{x} \mathrm{BiO}_{3}$ (BKBO) [15, 16]. We suppose that the double-well potential reflects strong electron-phonon coupling due to the dynamic charge inhomogeneity in both superconducting oxide classes: bismuthates and cuprates.

The goal of this paper is to specify the role of vibrations of oxygen ions in the appearance of the superconductive state using joint EXAFS data analysis of the local structure peculiarities in $\mathrm{Ba}_{1-x} \mathrm{~K}_{x} \mathrm{BiO}_{3}$ and in electron-doped $\left(\mathrm{Nd}_{1.85} \mathrm{Ce}_{0.15} \mathrm{CuO}_{4-\delta}\right)$ and hole-doped $\left(\mathrm{La}_{1.85} \mathrm{Sr}_{0.15} \mathrm{CuO}_{4}\right)$, to connect the local and the macroscopic properties of compounds and to make clear the role of electron-phonon coupling in the mechanism of high temperature superconductivity.

\section{Experiment}

Forboth $\mathrm{Ba}_{1-x} \mathrm{~K}_{x} \mathrm{BiO}_{3}(x=0,0.4,0.5)$ and $\mathrm{Nd}_{2-x} \mathrm{Ce}_{x} \mathrm{CuO}_{4-\delta}$ with $x=0,0.15,0.2$ (since $T^{\prime}$ structure has no apical oxygen atoms) we used polycrystalline ceramics synthesized by solid-state reaction technique. The $\mathrm{Nd}_{1.85} \mathrm{Ce}_{0.15} \mathrm{CuO}_{4-} \delta$ sample was annealed in reduced helium atmosphere for 10 hours at $1050{ }^{\circ} \mathrm{C}$ to achieve superconductive properties. To analyze correctly the EXAFS data inside the superconducting $\mathrm{CuO}_{2}$-plane of $T$-structure one should provide polarized measurements on single crystals since the $\mathrm{Cu}-\mathrm{O}(1)$ and $\mathrm{Cu}-\mathrm{O}(2)$ bond lengths inside and outside the plane are close and interfere in EXAFS spectra. High quality single crystal of $\mathrm{La}_{1.85} \mathrm{Sr}_{0.15} \mathrm{CuO}_{4}$ of $4 \times 2 \times 7 \mathrm{~mm}^{3}$ dimensions was prepared by a non-crucible floating zone melting method with radiation heating.

The X-ray absorption spectra above the $L_{3}-\mathrm{Bi}$ and $K-\mathrm{Cu}$ edges were collected at the at D-21 line (XAS-13) of DCI (LURE, Orsay, France) and E4 beamline of the DORIS III (DESY, Hamburg, Germany) storage rings respectively. Energy resolution of the double-crystal Si (111) monochromators with a $0.3 \mathrm{~mm}$ slit was about $1.4 \mathrm{eV}$ at $9 \mathrm{keV}$ and $2-3 \mathrm{eV}$ at $13 \mathrm{keV}$. Low-temperature measurements were carried out using a liquid-helium continuous flow cryostat with a temperature control of $\pm 1 \mathrm{~K}$ at $300 \mathrm{~K}$ and $0.1 \mathrm{~K}$ at $5 \mathrm{~K}$. Transmission spectra for $\mathrm{BKBO}$ and $\mathrm{NCCO}$ were measured on a pressed polycrystalline samples with the optimal thickness. Polarized fluorescence LSCO spectra were measured by 7-pixel $\mathrm{Si}(\mathrm{Li})$ detector on a single crystal sample placed in the sample holder in a way to obtain $E \| \mid a b$ polarization with $45^{\circ} \mathrm{X}$-ray incidence angle.

We analysed the EXAFS function $\chi(k)$, using the VIPER [17] software package by construction of the model potentials of atomic vibrations, subsequent calculation of the pair radial distribution function, and calculation of the model $\chi(k)$.

\section{Results}

As we pointed out earlier the generally accepted harmonic approach for treating EXAFS spectra cannot be applied both for superconducting compositions of BKBO $(x=0.4,0.5)[15,16]$ and for $\mathrm{CuO}_{2}$-plane of LSCO, NCCO $(x=0.15)[9,18]$ due to the strongly nonharmonic vibrations of oxygen ions. Thus we must use the general formula for EXAFS-function simulation, based on the pair radial distribution function $g_{\alpha \beta}(r)(\mathrm{PRDF})$ :

$$
\chi(k)=-S_{0}^{2} \frac{1}{k} \sum_{\alpha}\left|f_{\alpha}(\pi, k)\right| \int_{0}^{\infty} g_{\alpha \beta}(r) \sin \left[2 k r+\varphi_{\alpha}(k)\right] / r^{2} \mathrm{~d} r
$$

and find $g_{\alpha \beta}(r)$ by solving the stationary Schrödinger equation. As a result of correct EXAFS function simulation we obtain the parameters of the none-harmonic potential for oxygen ion vibrations. Let us consider a nature of this potential.

As mentioned above, for the superconductive compound $\mathrm{Ba}_{0.6} \mathrm{~K}_{0.4} \mathrm{BiO}_{3}$ the EXAFS- function was successfully simulated when a double-well potential was used to describe the oxygen vibrations $[15,16,19]$. Due to the strong covalence of $\mathrm{Bi} 6 s-\mathrm{O} 2 p_{\sigma}$ bonds the octahedral complexes $\mathrm{BiO}_{6}$ representing the most tightly bound items of the perovskite-like structure can be considered as quasimolecules [20]. It was supposed that the reason for appearance of a double-well potential is the difference in electron filling of the neighbouring octahedral complexes. Part of the octahedra denoted as $\mathrm{BiL}^{2} \mathrm{O}_{6}$ have a hole pair $\mathrm{L}^{2}$ in the upper antibonding molecular orbital $\overline{\mathrm{Bi}} 6 s-\mathrm{O} 2 p_{\sigma^{*}}$. They are characterized by stiff (quasi-molecular) $\mathrm{Bi}-\mathrm{O}$ bonds and a smaller radius. Rest of the $\mathrm{BiO}_{6}$ octahedra bearing electron pair represent non-stable molecules with the filled upper antibonding orbital. They have more soft $\mathrm{Bi}-\mathrm{O}$ bonds and a larger radius. The equilibrium position of oxygen ions belonging to neighbouring octahedra of different kinds is slightly displaced from the middle point between two $\mathrm{Bi}$ sites to bismuth position in the small $\mathrm{BiL}^{2} \mathrm{O}_{6}$ octahedron. Local hole and electron pairs can exchange each other due to tunneling from one complex to another. As a result the oxygen ion belonging to two octahedra of different kinds vibrates non-harmonically because the equilibrium position of the vibration potential oscillates in accordance with the dynamic exchange $\mathrm{BiO}_{6} \leftrightarrow \mathrm{BiL}^{2} \mathrm{O}_{6}$. Such vibrations were described by a double-well potential with a high transparency of a barrier separating the wells $[15,16,19]$.

By analogy with the description above we successfully simulated EXAFS-function of the first $\mathrm{Cu}-\mathrm{O}(1)$ shell inside $\mathrm{CuO}_{2}$-plane according to the formula (1) using double-well oscillation potential for both $\mathrm{Nd}_{1.85} \mathrm{Ce}_{0.15} \mathrm{CuO}_{4-\delta}$ and $\mathrm{La}_{1.85} \mathrm{Sr}_{0.15} \mathrm{CuO}_{4}$ superconducting compounds (see Fig. 1, left panels, dotted lines). In hole-doped LSCO there are two types of octahedral complexes: $\mathrm{CuL}^{1} \mathrm{O}_{6}$ with half-filled upper antibonding molecular orbital $\overline{\mathrm{Bi}} 6 s-\mathrm{O} 2 p_{\sigma^{*}}$ and $\mathrm{CuL}^{2} \mathrm{O}_{6}$ complexes, where the above orbital has hole pair $\mathrm{L}^{2}$. The electron-doped NCCO compound, which has no apical oxygen, represents a structure of square complexes: $\mathrm{CuL}^{1} \mathrm{O}_{4}$ with half-filled upper antibonding molecular orbital $\mathrm{Bi} 6 s-\mathrm{O} 2 p_{\sigma^{*}}$ and $\mathrm{CuO}_{4}$ complexes, where the above orbital has an electron pair. We supposed that the oxygen ions bounding complexes of different kinds oscil- 
late in a double-well potential and the ions belonging to identical $\mathrm{CuL}^{1} \mathrm{O}_{n}(n=4,6)$ complexes oscillate in ordinary harmonic potential. To construct the double-well potential we considered a parabolic form for each well, $U_{1}=\kappa_{1}\left(r-r_{1}\right)^{2} / 2$ and $U_{2}=\kappa_{2}\left(r-r_{2}\right)^{2} / 2$, joined continuously. In our calculations we assumed that amount of oxygen ions oscillating in double-well potential is determined by the amount of doping holes or electrons and is equal to $15 \%$. The rest ions oscillate in the single-well parabolic potential. Results of the calculations presented in the Fig. 1 (left panels, dotted lines) demonstrate good simulation of the model EXAFS-function to the experimental one in whole $k$-range.

The $\mathrm{La}_{2} \mathrm{CuO}_{4}$ and $\mathrm{Nd}_{2} \mathrm{CuO}_{4}$ parent compounds represent the structure from identical $\mathrm{CuL}^{1} \mathrm{O}_{n}(n=4,6)$ complexes with the half-filled upper antibonding molecular orbital $\mathrm{Cu} 3 d-\mathrm{O} 2 p_{\sigma^{*}} . \mathrm{La}_{2} \mathrm{CuO}_{4}$ and $\mathrm{Nd}_{2} \mathrm{CuO}_{4}$ are the Motttype insulators (see left part of upper and lower panels in Fig. 2), since the charge transfer from one complex to a neighbouring one entails energy expense (activation energy $E_{a} \sim 2 \mathrm{eV}$ ) because it leads to the change of the electronic structure of both complexes. Presence of unpaired spins in the $\mathrm{CuL}^{1} \mathrm{O}_{n}$ complexes determines the antiferromagnetic ground state of the compounds.

When part of lanthanum is replaced by strontium the $\mathrm{CuL}^{2} \mathrm{O}_{6}$ octahedral complexes with two free states at the upper antibonding orbital are formed (see Fig. 2, center) since the $\mathrm{Sr}^{+2}$ ion provides one electron less than the
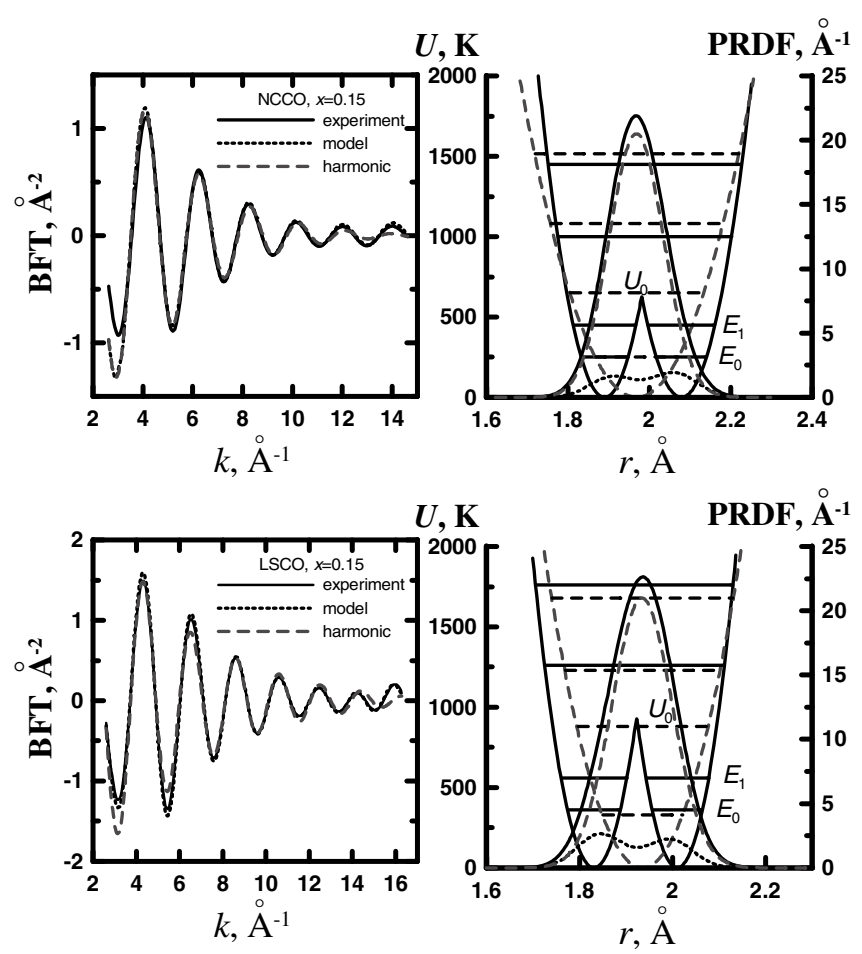

Fig. 1. Left panels: the experimental (solid) and model (dotted lines) EXAFS-function $\chi(k) k^{2}$ of the first $\mathrm{Cu}-\mathrm{O}(1)$ shell measured at $10 \mathrm{~K}$ for $\mathrm{Nd}_{1.85} \mathrm{Ce}_{0.15} \mathrm{CuO}_{4-\delta}$ (upper panel) and $\mathrm{La}_{1.85} \mathrm{Sr}_{0.15} \mathrm{CuO}_{4}$ (lower panel); dashed lines showed the fitting in harmonic approach. Right panels: the model potential (harmonic and double-well) with the corresponding PRDF's and energy levels $E_{0}, E_{1}, \ldots$ The total PRDF is shown by a solid line, while the partial PRDF's are shown by dashed line for harmonic potential and by dotted line - for double-well potential. $U_{0}$ is the energy of the potential barrier.
$\mathrm{La}^{+3}$ ion. Replacement of a part of neodymium by cerium leads to appearance of the $\mathrm{CuO}_{4}$ complexes with fullyfilled electronic states at the upper antibonding orbital since the $\mathrm{Ce}^{+4}$ ion provides one electron greater than the $\mathrm{Nd}^{+3}$ ion. So the doping of $\mathrm{La}_{2} \mathrm{CuO}_{4}$ and $\mathrm{Nd}_{2} \mathrm{CuO}_{4}$ provides local pairs i.e. the $\mathrm{CuL}^{2} \mathrm{O}_{6}$ complexes, containing the hole pair and the $\mathrm{CuO}_{4}^{-}$complexes, containing the electron pair. Tunneling of electron from the complex to the free state of the neighboring one leads to replacement of them by each other. The pairs are transferred into the neighboring complex under the tunneling so the dynamic exchange results in the local pair movement. At the same time only single charge is transferred at this movement.

Local hole (electron) pairs can move between neighbouring complexes in compliance with the vibrations of oxygen ions in a double-well potential due to the dynamic exchange $\mathrm{CuL}^{1} \mathrm{O}_{6} \leftrightarrow \mathrm{CuL}^{2} \mathrm{O}_{6}\left(\mathrm{CuO}_{4} \leftrightarrow \mathrm{CuL}^{1} \mathrm{O}_{4}\right)$. At low temperatures the movement of the local pairs becomes coherent and this explains the appearance of the superconductive state (see center of the both panels in Fig. 2).

It is worth to notice, that the local structure picture, presented in Fig. 2, is very closed to ideas, proposed by K. A. Müller and J. G. Bednorz in their Nobel prize lecture in 1987 [21].

In the overdoped regime $(x>0.28)$ for LSCO the concentration of hole pairs becomes too large for the wave function of the pair to have local character and free electronic levels of $\mathrm{CuL}^{2} \mathrm{O}_{6}$ complexes delocalize. These levels split and overlap with half-filled $\underline{\mathrm{L}}^{1}$ levels of $\mathrm{CuL}^{1} \mathrm{O}_{6}$ complexes. As a result there is formed a partly (less than half) filled conductivity band. So the hole doping of initial $p$-type semiconductor $\mathrm{La}_{2} \mathrm{CuO}_{4}$ converts it to a regular $n$-type metal in the overdoped regime (see Fig. 2, upper panel, right).

In case of $\mathrm{NCCO}$ in the overdoped regime $(x>0.18)$ electrons from fully occupied levels of $\mathrm{CuO}_{4}$ complexes delocalize and together with half-filled levels of $\mathrm{CuL}^{1} \mathrm{O}_{4}$ complexes create the band with more than half filling, producing the hole-type conductivity in such a system (see Fig. 2, low panel, right). So the electron doping of $n$-type parent semiconductor $\mathrm{Nd}_{2} \mathrm{CuO}_{4}$ converts it to $p$-type metal in the overdoped regime.

In our phenomenological description we assume that the local electron (hole) pairs appear in $\mathrm{CuO}_{n}(n=4,6)$ complexes as a result of doping of the parent insulators $\mathrm{Nd}_{2} \mathrm{CuO}_{4}\left(\mathrm{La}_{2} \mathrm{CuO}_{4}\right)$ by $\mathrm{Ce}(\mathrm{Sr})$. The crucial role of the lattice vibrations (phonon subsystem) in our model lays in providing the phase stiffness, i.e. the ability of phase coherence in the local pair moving at $T<T_{c}$. We suppose that this coherency at low temperature is provided by the peculiarities of the perovskite-like structure. As we pointed out for BKBO system [16, 19] in the superconducting perovskite-like oxides there exists a soft collective rotational mode of $\mathrm{BiO}_{6}\left(\mathrm{CuO}_{n}\right.$ complexes $)$, that does not freeze at low temperature [22]. So the distance between the minima of the double-well potential is modulated by this collective rotational mode and as a sequence the frequency of oxygen ion tunneling between the potential wells is bounded by frequency of a soft rotational mode or its harmonics.

At low temperature the probability of tunneling between the two wells reaches its maximum when oxygen 


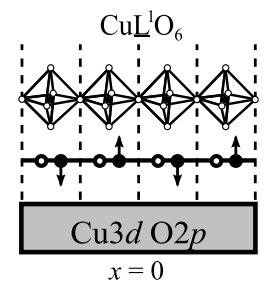

AFM insulator

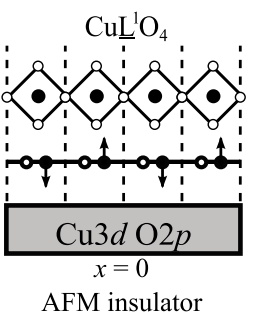

$\mathrm{CuL}^{2} \mathrm{O}_{6} \quad \mathrm{CuL}^{2} \mathrm{O}_{6} \quad \mathrm{CuL}^{2} \mathrm{O}_{6}$
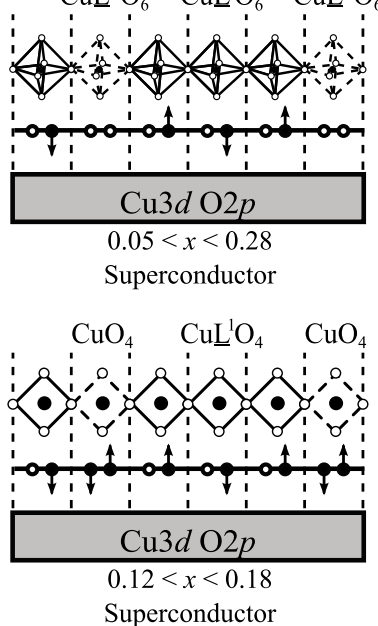
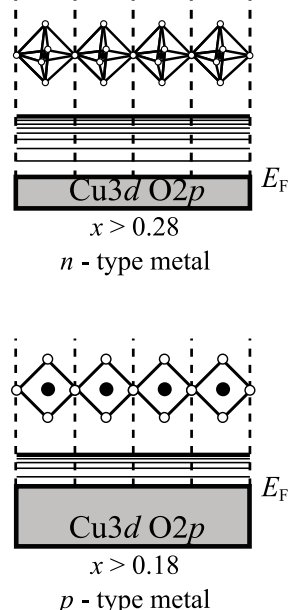

Fig. 2. Scheme of the insulator-metal phase transition for $\mathrm{La}_{2-x} \mathrm{Sr}_{x} \mathrm{CuO}_{4}$ (upper panel) and for $\mathrm{Nd}_{2-x} \mathrm{Ce}_{x} \mathrm{CuO}_{4-\delta}$ (lower panel). The local crystalline structure of the $\mathrm{CuO}_{n}(n=4,6)$ complexes (at the top of both panels) and the local electronic structure (at the bottom of panels) are shown. The occupied states of the $\mathrm{Cu} 3 d-\mathrm{O} 2 p$ valence band are marked in grey. Black and white circles denote electrons and holes, respectively. On the left of both panels the antiferromagnetic phase of the parent insulators $\mathrm{La}_{2} \mathrm{CuO}_{4}$ and $\mathrm{Nd}_{2} \mathrm{CuO}_{4}$ is shown. The superconductive phase of $\mathrm{La}_{2-x} \mathrm{Sr}_{x} \mathrm{CuO}_{4}$ with $0.05<x<0.28$ and of $\mathrm{Nd}_{2-x} \mathrm{Ce}_{x} \mathrm{CuO}_{4-\delta}$ superconductive state $(0.12<x<0.18)$ is presented in the center. The metal state with Fermi level $E_{F}$ in overdoped regime is shown on the right: the $n$-type metal for LSCO at $x>0.28$ (upper panel) and the $p$-type metal for NCCO at $x>0.18$ (lower panel).

ions intersect $\mathrm{Cu}-\mathrm{Cu}(\mathrm{Bi}-\mathrm{Bi})$ directions due to vibrations in the collective rotational mode of $\mathrm{CuO}_{n}\left(\mathrm{BiO}_{6}\right)$ complexes, which guarantees the phase coherence between the vibration in the double-well potential and the vibration in the half-breathing mode (see Fig. 3). This provides coherence of the local pair movement, i.e. the ability of the superconducting state to carry a supercurrent. When temperature increases higher than the critical temperature the probability of tunneling increases and oxygen ions can tunnel at any other moment than the moment when they cross [100]-type axes. It destroys the phase coherence between the oxygen ion vibrations in double-well potential and in half-breathing mode. As a result the phase coherence of the local pair moving is destroyed and superconductivity disappears.

Here we should point out that the peculiar role of oxygen ion vibrations in HTSC mechanism was discussed in a long list of papers (see the review article [23] and references therein).

As it was pointed above, the double-well potential was independently observed in other superconducting cuprates $\mathrm{La}_{2} \mathrm{CuO}_{4.1}[7,10]$, which indicates that anomalous oxygen ion vibration in a double-well potential is an inherent property of superconductive oxides with perovskite-like structure. Meanwhile the authors of $[7,10]$ explain the appearance of the double-well potential in terms of JahnTeller polaron model. Their model is inapplicable for $\mathrm{Nd}_{1.85} \mathrm{Ce}_{0.15} \mathrm{CuO}_{4-\delta}$ because of the absence of the apical

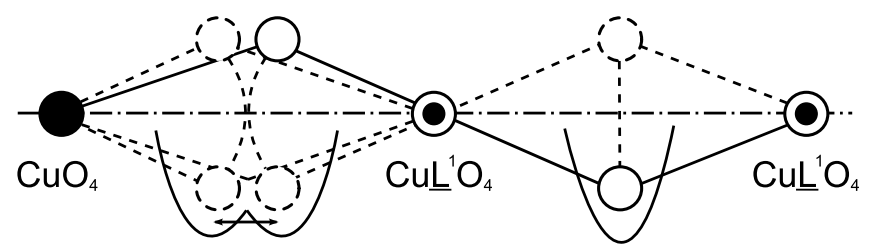

Fig. 3. A sketch of the oxygen ion vibrations in $\mathrm{Nd}_{2-x} \mathrm{Ce}_{x} \mathrm{CuO}_{4-\delta}$ in the case of different (left) and equivalent (right) neighbouring complexes with corresponding radial oscillatory potentials. oxygen atoms in $\mathrm{NCCO}$ structure and for BKBO with cubic structure. So we suppose that our phenomenological description of the relationship between local electronic and local crystalline structures is more suitable for explaining the appearance of the superconductive state in bismuthates and in cuprates irrespective of the type of doping.

\section{Conclusion}

The joint EXAFS data analysis of the local structure peculiarities in $\mathrm{Ba}_{1-x} \mathrm{~K}_{x} \mathrm{BiO}_{3}, \quad \mathrm{Nd}_{1-x} \mathrm{Ce}_{x} \mathrm{CuO}_{4-\delta}$ and $\mathrm{La}_{1.85} \mathrm{Sr}_{0.15} \mathrm{CuO}_{4}$ allowed us to observe the correlations between the local and macroscopic properties and to make conclusions that bismuthates and both electron- and holedoped HTSC cuprates reveal similar local dynamic lattice instability which is manifested as vibrations of part of oxygen ions in the double-well potential and these abnormal vibrations occur due to different electronic filling of neighbouring $\mathrm{BiO}_{6}$ and $\mathrm{CuO}_{n}(n=4,6)$ complexes.

Proposed phenomenological description of the relationship of the electronic and local crystalline structures explains the insulator-to-metal phase transition and the appearance of the superconductive state with both hole or electron doping of parent insulators and clarifies the role of electron-phonon coupling in superconductivity.

Acknowledgments. We acknowledge the LURE and HASYLAB Program Committees for providing beamtime, G. H. Panova and A. M. Balbashev for providing the LSCO single crystals. This work was supported by Russian Foundation for Basic Researches (grants 08-0200759-a and 09-02-12257-ofi_m) and was done in the framework of the Federal Program "Scientific and Scientific-Pedagogical Staff of Innovative Russia" 2009-2013.

\section{References}

[1] Sleight, A. W.; Gillson, J. L.; Bierstedt, P. E.: Solid State Commun. 17 (1975) 27.

[2] Bednorz, J. G.; Müller, K. A.: Z. Phyz.: Condens. Vatter 64 (1986) 189. 
[3] Newns, D. M.; Tsuei, C. C.: Nature Physics 3 (2007) 184.

[4] Reznik, D.; Sangiovanni, G.; Gunnarsson, O.; Devereaux, T. P.: Nature 455 (2008) E6.

[5] Iwasawa, H.; Douglas, J. F.; Sato, K.; Masui, T.; Yoshida, Y.; Sun, Z.; Eisaki, H.; Bando, H.; Ino, A.; Arita, M.; Shimada, K.; Namatame, H.; Taniguchi, M.; Tajima, S.; Uchida, S.; Saitoh, T.; Dessau, D. S.; Aiura, Y.: Phys. Rev. Lett. 101 (2008) 157005.

[6] Bianconi, A.; Saini, N. L.; Lanzara, A.; Missori, M.; Rossetti, T.; Oyanagi, H.; Yamaguchi, H.; Oka, K.; Ito, T.: Phys. Rev. Lett. 76 (1996) 3412-5.

[7] Bishop, A. R.; Mihailovic, D.; Mustre de Leon, J.: J. Phys.: Cond. Matt. 15 (2003) L169.

[8] Saini, N. L.; Oyanagi, H.; Scagnoli, V.; Ito, T.; Oka, K.; Bianconi, A.: Europhys. Lett. 63 (2003) 125.

[9] Menushenkov, A. P.: J. Synchrotron Rad. 10 (2003) 369.

[10] Mustre de Leon, J.; Acosta-Alejandro, M.; Conradson, S. D.; Bishop, A. R.: J. Synchrotron Rad. 12 (2005) 193.

[11] Oyanagi, H.; Zhang, C.; Tsukada, A.; Naito, M.: Journal of Physics: Conference Series 108 (2008) 012038

[12] Zhang, C. J.; Oyanagi, H.: Phys. Rev. B 79 (2009) 064521.

[13] Bussmann-Holder, A.; Bishop, A. R.: Phys. Rev. B 51 (1995) 3246-9.

[14] Orenstein, J.; Millis, A. J.: Science 288 (2000) 468

[15] Menushenkov, A. P.; Klementev, K. V.; Konarev, P. V.; Meshkov, A. A.: JETP Letters 67 (1998) 1034.
[16] Menushenkov, A. P.; Klementev, K. V.: J. Phys.: Cond. Matt. 12 (2000) 3767.

[17] Klementiev, K. V.: VIPER for Windows, freeware www.cells.es/ Beamlines/CLAESS/software J. Phys. D: Appl. Phys. 34 (2001) 209.

[18] Menushenkov, A. P.; Chernikov, R. V.; Ivanov, A. A.; Sidorov, V. V.; Klementiev, K. V.: Journal of Physics: Conference Series 190 (2009) 012093.

[19] Menushenkov, A. P.; Klementev, K. V.; Konarev, P. V.; Meshkov, A. A.; Benazeth, S.; Purans, J.: Nuclear Instruments and Methods in Physics Research A448 (2000) 340.

[20] Sugai, S.: Jpn. J. of Appl. Phys Suppl. 26-3-2 26 (1987) 1123.

[21] Bednorz, J. G.; Müller, K. A.: Perovskite-Type Oxides - The New Approach to High- $T_{c}$ Superconductivity. In: Nobel Lectures, Physics 1981-1990 (Ed. T. Frängsmyr, G. Ekspảng), p. 424. (World Scientific, Singapore) 1993.

[22] Markert, J. T.; Dalichaouch, Y.; Maple, M. B.: Rare Earth and Other Substitutions in High Temperature Oxide Superconductors. In: Physical Properties of High Temperature Superconductors (Ed. D. M. Ginsberg), Vol. 1, p. 265. (World Scientific, New York) 1989.

[23] Keller, H.; Bussmann-Holder, A.: Local Electron-Lattice Interactions in High-Temperature Cuprate Superconductors. In: Advanced in Condensed Matter Physics (Ed. A. Alexandrov), Vol. 2010, 393526. (Hundawi Publishing Corporation) 2010. 